\title{
Van Heel's coma lenses
}

Kees Smorenburg

Kees Smorenburg, "Van Heel's coma lenses," Proc. SPIE 3190, Fifth International Topical Meeting on Education and Training in Optics, (8 December 1997); doi: 10.1117/12.294415

Event: Fifth International Topical Meeting on Education and Training in Optics, 1997, Delft, Netherlands 


\title{
Van Heel's Coma Lenses
}

\author{
C. Smorenburg \\ + TNO Institute of Applied Physics, P.O. Box 155.2600 AD Delft, The Netherlands
}

\begin{abstract}
In education in optics the explanation and demonstration of aberrations are an essential part. Of the 3rd order monochromatic aberrations usually most attention is paid to spherical aberration, astigmatism, field curvature and distortion. Coma is more complex to explain and more difficult to demonstrate in its pure form. In 1933 Van Heel has designed a "lens with pure coma" for demonstration purposes.

With this lens a number at photographs has been made to show the development of the coma circle from the pupil plane (single circle) to the image plane (double circle) and to demonstrate the coma images around the focal plane.

In 1948 a 2nd lens for demonstration of coma was made by Van Heel. Both lenses are described in this paper and their performance is determined by computer calculations. Comparison with original coma photographs is made.
\end{abstract}

Keywords: aberrations, coma

\section{INTRODUCTION}

For many optical activities in the area of design and instrumentation a good insight in the nature of aberrations is necessary. Concerning the 3rd order aberrations there is much attention for spherical aberration, astigmatism, field curvature and even distortion. These aberrations are rather easy to explain and demonstrate in an almost pure shape. Concerning coma, this aberration is more difficult to explain (e.g. the double circle) and especially its appearance in most optical systems is less dominant.

Demonstration of pure coma with "standard" optics is mostly not convincing because the presence of the other aberrations like spherical aberration or astigmatism are disturbing the effect. The immediate cause of my interest for this coma aberration is a number of aberration photographs, that originally have been made by A.C.S. van Heel in 1934.

These separate pictures have been mounted in a frame, that is in my possession. A copy of this frame is presented in figure 1 .

The collection of aberration photographs was used in the past to explain to students the character of the 3rd order aberration of coma. To make the pictures a special lens have been designed with almost negligible spherical aberration and astigmatism and a "huge" 3rd order coma. This lens is described in ref. 1.

In 1933 R. Kingslake and A.B. Simmons published the results of calculations concerning the influence of coma and astigmatism on the imaging cone near the focal plane (ref.2). They calculated the theoretical cross sections of the image cone in the presence of coma and astigmatism. Cone cross sections are given for pure coma, 3 parts coma +1 part astigmatism , 2 parts coma +2 parts astigmatism , 1 part coma +3 parts astigmatism and finally for pure astigmatism .

At that time 3rd order aberrations, especially coma were an interesting topic.

In this article the original coma lens of Van Heel is considered in some more detail. Especially the measurements of Van Heel are compared with present day computer calculations (spot diagrams). 


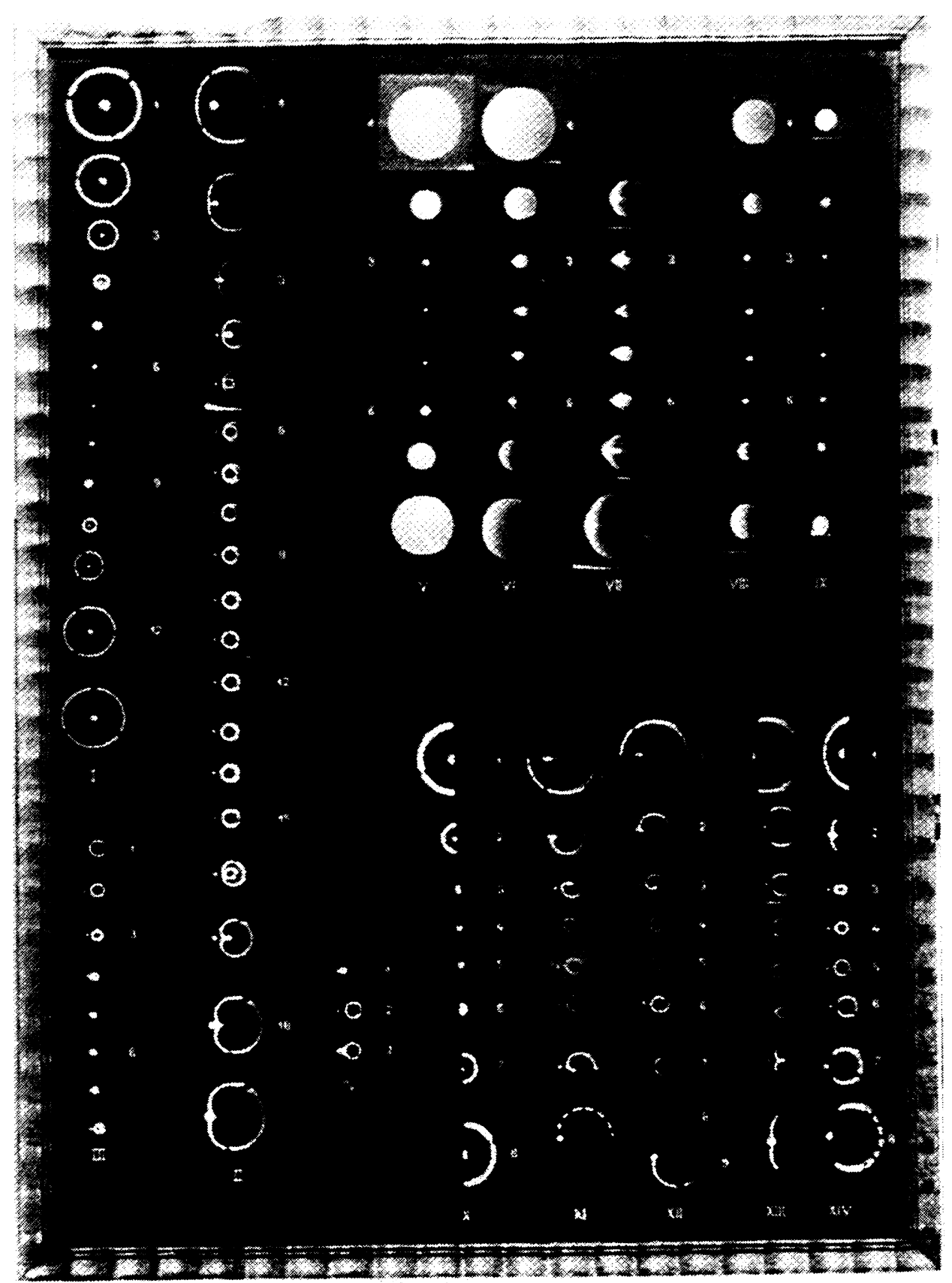

Figure 1: The frame with the original photographs of aberration, as made with the first coma lens of Van Heel in 1934. 


\section{COMA}

Coma is one of the five monochromatic 3rd order optical aberrations.

According to aberration theory the transverse deviation of rays in the paraxial image plane is given by:

(e.g.ref.2)

$$
\begin{gathered}
x^{\prime}=-\left(A \rho^{3} \sin \varnothing+B \rho^{2} h \sin 2 \varnothing+C \rho h^{2} \sin \varnothing+D \rho h^{2} \sin \varnothing\right) \\
y^{\prime}=-\left(A \rho^{3} \cos \varnothing+B \rho^{2} h(2+\cos 2 \varnothing)+3 C \rho h^{2} \cos \varnothing+D \rho h^{2} \cos \varnothing+E h^{3}\right)
\end{gathered}
$$

where the co-ordinates of a ray in the exit pupil is given by $\rho$ (radius) and $\emptyset$ (angle) and where $h$ is the paraxial height in the image plane.

The factors A, B, C, D and E are the so called aberration coefficients.

Here $A$ is the aberration coefficient of spherical aberration, B of coma, C of astigmatism, D of field curvature and $\mathrm{E}$ of distortion.

While playing with these coefficients from e.g. (1) and (2) the various aberrations can be derived rather simple.

For coma we have $\mathrm{A}=\mathrm{C}=\mathrm{D}=\mathrm{E}=0$, so

$$
\begin{gathered}
x^{\prime}=-B \rho^{2} h \sin 2 \varnothing \\
y^{\prime}=-B \rho^{2} h(2+\cos 2 \varnothing)
\end{gathered}
$$

Eliminating $\varnothing$ gives:

$$
x^{\prime 2}+\left(y^{\prime}-2 R\right)^{2}=R^{2}
$$

where $R=B \rho^{2} h$

Looking to equation (5) it is clear, that coma is built up of circles (with radius $\mathrm{R}$ ). The radius of the circle is linear proportional to the coma aberration coefficient $B$, linear proportional to the off-axis location $\mathrm{h}$ (so to the field angle) and quadratic proportional to the pupil radius $\rho$ (so to the aperture). The shift of the circle is $2 R$, the diameter of the circle.

From this a coma image can be constructed easily (figure 2). Also the topangle of 60 degrees is made clear in this way.

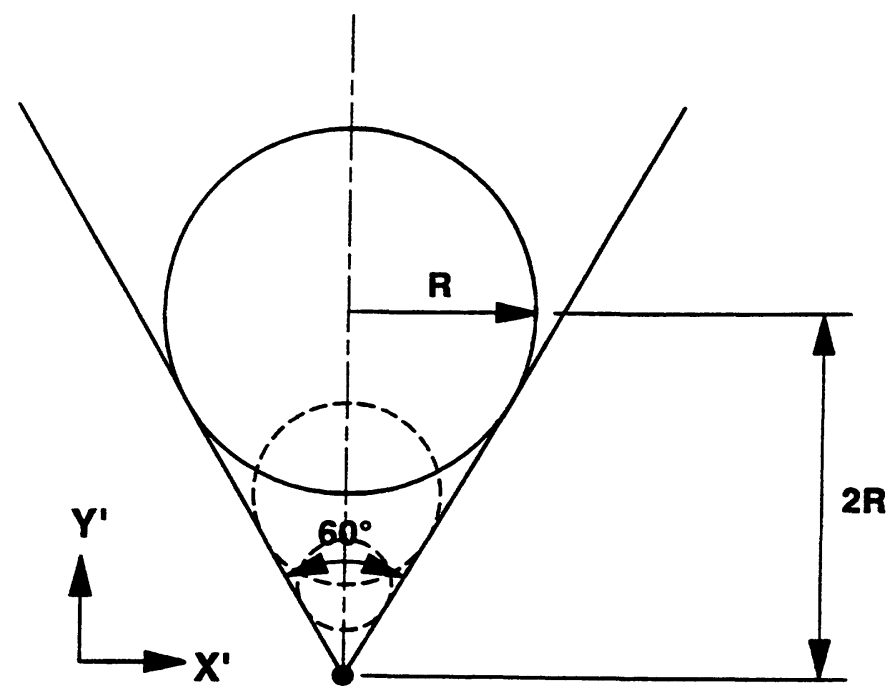

Figure 2: Construction of coma image 
An interesting feature of coma is the term $2 \varnothing$ in equation (3) and (4). This means, that half a circular segment in the exit pupil gives a full circular image in the focal plane. In between the exit pupil and the focal plane a metamorphosis takes place by changing the single circle into a double circle. The cross sections of the transit figures can be calculated with:

$$
\begin{gathered}
x "=(1-k) \sin 2 \varnothing+k \sin \varnothing \\
y^{\prime \prime}=(1-k)(2+\cos 2 \varnothing)+k \cos \varnothing
\end{gathered}
$$

Here for simplicity of calculation the factors $B, \rho$ and $h$ have been taken 1 .

The factor $\mathrm{k}$ indicates the relative position of the plane between exit pupil and focal plane.

Focal plane: $\mathrm{k}=0 \rightarrow$ Double (coma) circle.

Exit pupil: $\mathrm{k}=1 \rightarrow$ Single (pupil) circle.

With these simple formulas the transition from single - to double circle can be calculated. In figure 3 some calculated cone sections between exit pupil and focal plane are presented.
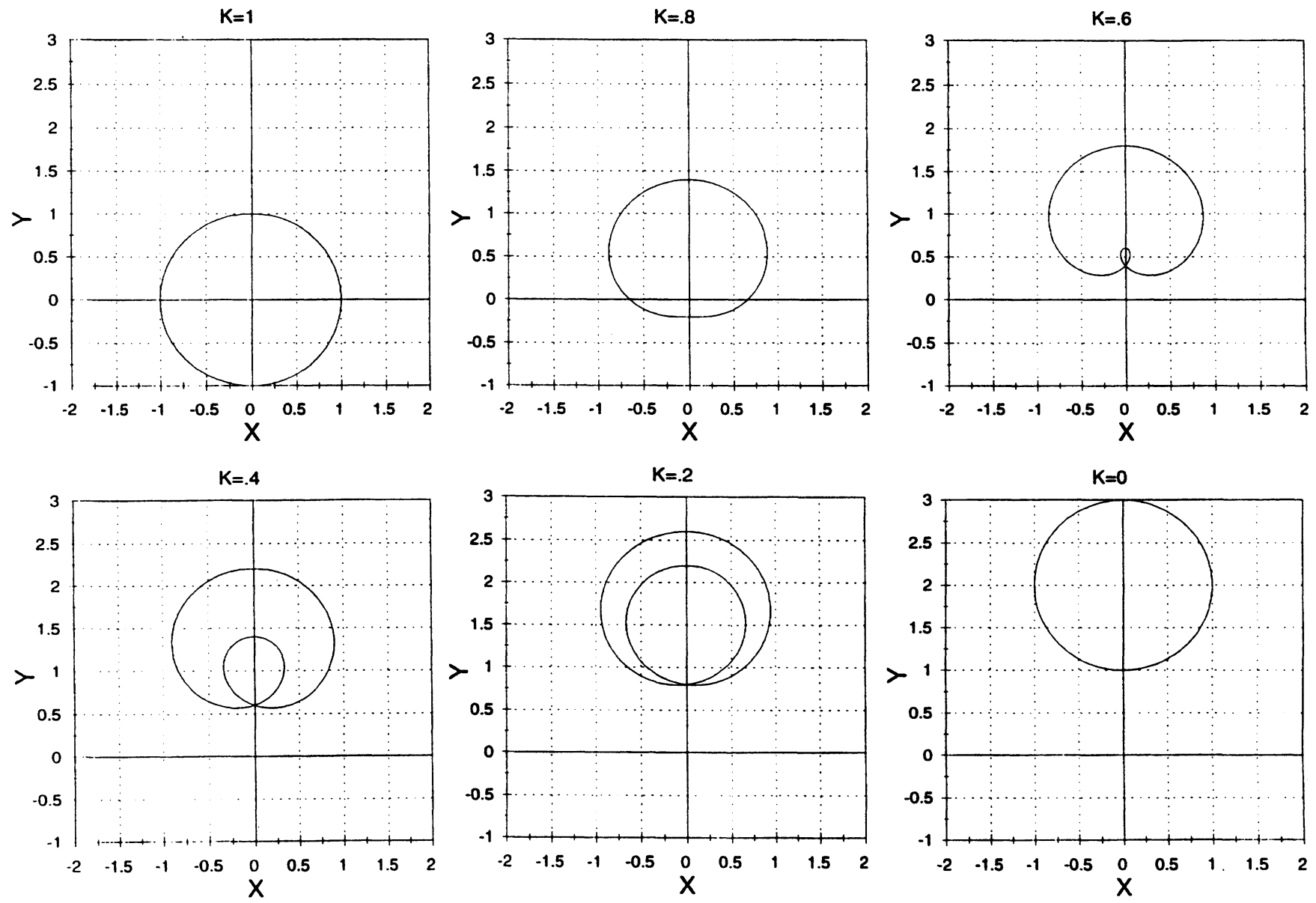

Figure 3: The development of the double coma circle, as calculated with equation (6) on (7)

$\mathrm{K}=1$ single circle in the exit pupil

$\mathrm{K}=0$ double circle in the focal plane 


\section{THE FIRST COMA LENS}

To demonstrate especially the coma aberration a special lens configuration is designed and manufactured. The lens consists of 2 components (crown + flint). The relative aperture F/27 ensures, that $3 \mathrm{rd}$ order aberrations are dominating the image and that the influence of higher order aberrations can be neglected.

The optical data of the lens are:

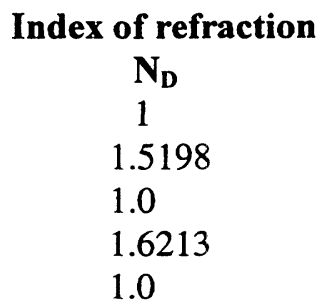

$\begin{array}{cc}\text { Radius } & \text { Thickness } \\ \text { R (mm) } & \text { T (mm) } \\ +247.16 & 4.53 \\ -367.34 & 0.12 \\ +189.03 & 5.805 \\ +103.59 & \end{array}$

The indices of refraction are given for the sodium line $(\lambda=589 \mathrm{~nm})$.

The focal length is $1070 \mathrm{~mm}$ and the useful diameter is about $\varnothing=40 \mathrm{~mm}$.

The 3rd order aberrations of the lens have been calculated with the Seidel approximation for an object distance of $10 \mathrm{~m}$.

The size of the aberrations in the paraxial image plane is, using an entrance pupil with a diameter $\varnothing=$ $30 \mathrm{~mm}$ : and an off-axis angel of 13.3 degrees:

Spherical aberration: $0.06 \mathrm{~mm}$.

Coma (diameter of largest circle): $1.5 \mathrm{~mm}$.

Astigmatism (length of astigmatic line): $0.08 \mathrm{~mm}$.

From these figures it is clear, that coma is the dominant aberration .

The lens has been manufactured and to demonstrate the coma aberration an experimental arrangement has been made.

The object was a circular aperture with a diameter $\varnothing=2 \mathrm{~mm}$, which was illuminated by a sodium discharge tube $(\lambda=589 \mathrm{~nm})$. Just in front of the lens diaphragms could be positioned with special open areas.

A diaphragm with an open area with a diameter $\varnothing=32 \mathrm{~mm}$ was used to create the whole coma image, while another diaphragm with a ring shaped open area (diameter $\varnothing=30 \mathrm{~mm}$, thickness of ring 2.5 $\mathrm{mm}$ ) was used to demonstrate the generation of the (double) coma circle.The coma images were stored on a Kodak Super Pan film.

To study the development of an aberration pattern near the paraxial image plane pictures were taken in a number of planes around the paraxial plane.

The development of the coma aberration is shown in column II and column VII of figure 1 in a clear way. The pictures of column II show the intersection of the optical beam with 19 planes located around the focal plane.

The double coma circle is found somewhere at position 12 or 13.

A number of these cross sections have also been calculated with the optical program ZEMAX.

A random pupil filling mode was used to create spotdiagrams.

The results of these calculations are presented in figure 4. 


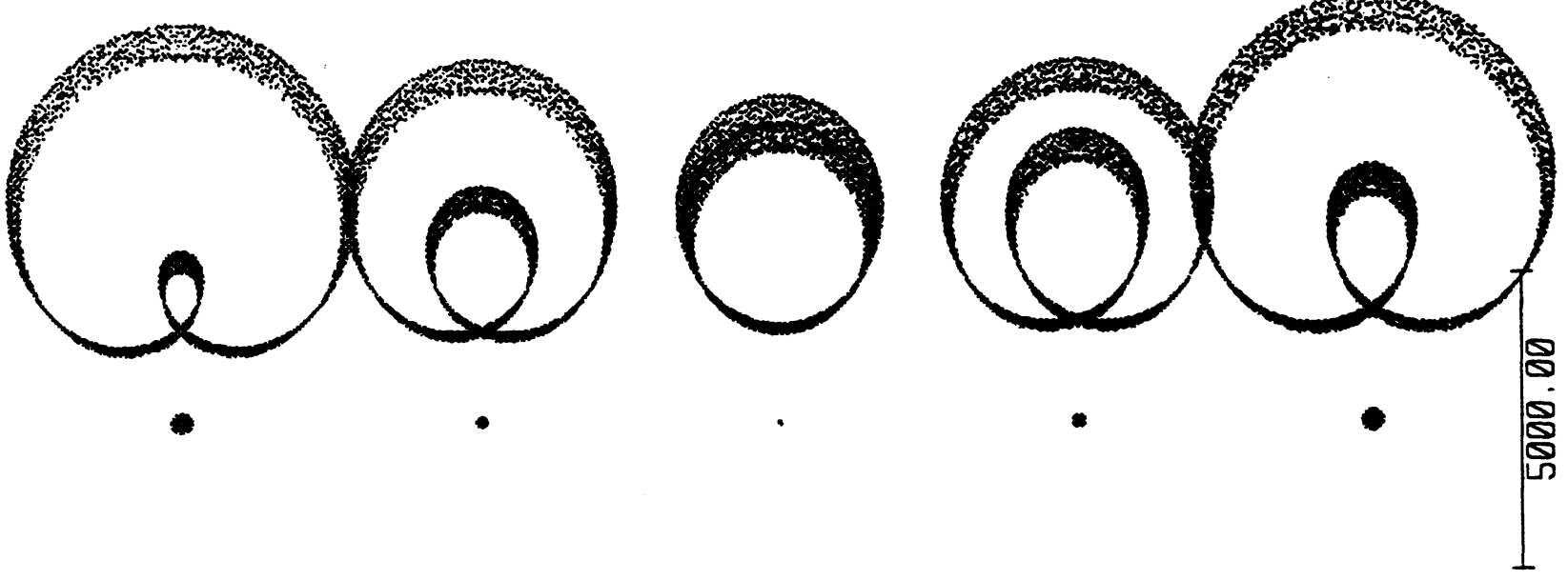

Figure 4: Calculated coma patterns of the first coma lens

These figures correspond with the positions II-4, II-5, II-12, II-16 and II-17 of figure 1 . The calculated spotdiagrams are based on pure geometrical ray-tracing not including diffraction effects.

This explains the differences in thickness of the rings. The pictures of column VII show the intersection of the full coma beam with 7 planes around the focal plane. Position VII- 4 shows the ideal coma image in the paxial image plane.

In figure 1 a number of other pictures are presented. For the explanation the interested reader is referred to ref.1.

Van Heel has used the same lens also for experiments to assess the contamination of various $3^{\text {rd }}$ order aberrations. For example spherical aberration can be added by rotating the lens over 180 degrees or by placing a thin uncorrected lens behind the coma lens.

Also beautiful pictures showing almost pure astigmatism have been made by combining the coma lens with a cylinder lens.

A more detailed description is given in ref.1.

\section{THE SECOND COMA LENS}

The lens, that is described in the previous section was designed and manufactured in 1933/1934. The pictures of aberrations, that have been made with this lens still exist, but the lens itself has been lost. In my possession however is another lens with the inscription: $\mathrm{f}=40.5 \mathrm{~cm}$, coma-lens waarn.bk. 12, 79-'49. Here "waarn.bk" means a hand-written book, which Van Heel used to describe ideas, inventions, designs, calculations etc.

This lens has a beautiful coma. The lens consists of 2 components of F8 and BK4. 
The data of the lens are:

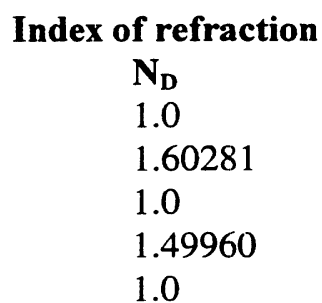

Radius
R(mm)
-47.92
-91.16
-821.5
-70.41

Thickness

$\mathbf{T}(\mathbf{m m})$

13.2

7.07

12.30

The diaphragm is located at $8.1 \mathrm{~mm}$ in front of the first lens surface.

All radii have a negative value (!).

The object is at infinity, the focal length is $405 \mathrm{~mm}$. In figure 5 the coma image of this lens at a field angle of 20 degrees is given. The diaphragm has a diameter $\varnothing=40 \mathrm{~mm}$ (F/10 aperture). The calculations have been performed with ZEMAX.

The dimension of the largest coma circle is about $2 \mathrm{~mm}$.

Figure 6 shows a picture of the coma spot.

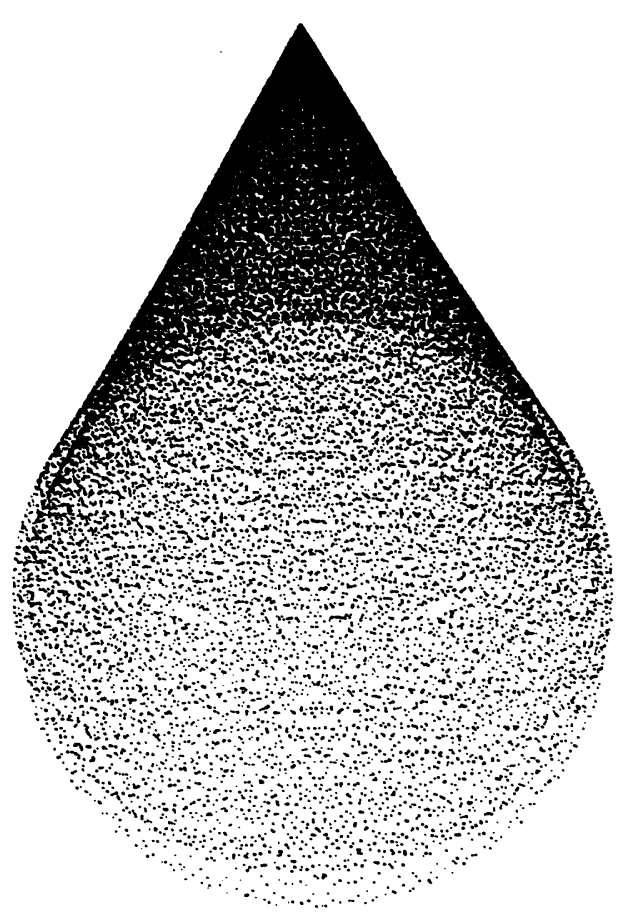

Figure 5: Computer coma image

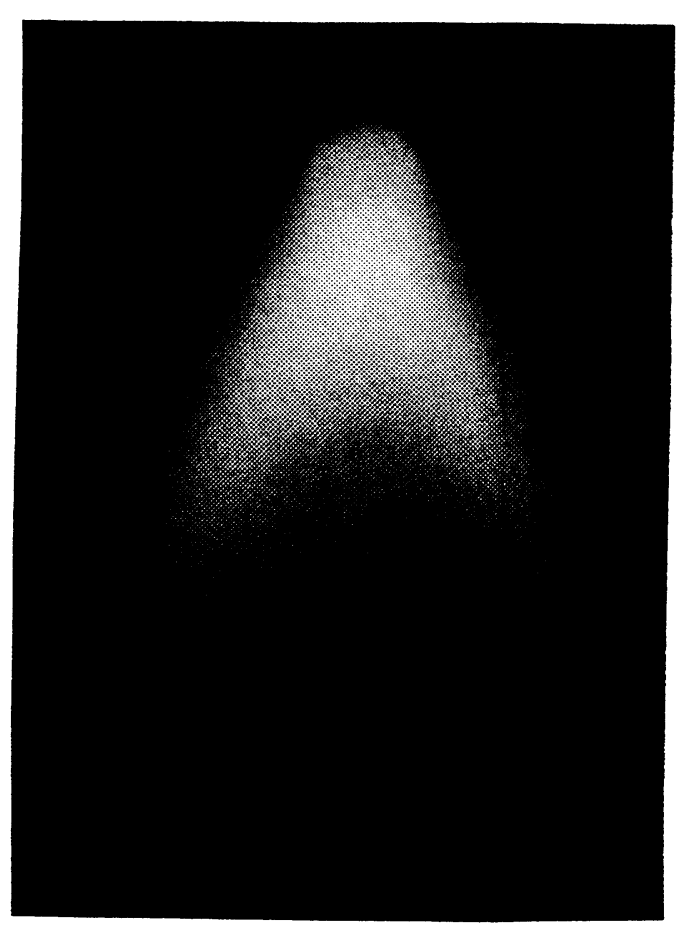

Figure 6: Photograph of coma aberration

\section{CONCLUDING REMARKS}

In this paper 2 lenses of Van Heel for the demonstration of coma are described. From the first lens (from 1934) the coma pictures still exist and can be used for an instructive demonstration of coma in and around the focal plane.

The optical data of the lens are known, which makes a remanufacturing (if desired) possible. Present computer calculations have confirmed the original coma pictures.

The second coma lens (from 1948) still exists and can be used for demonstration. 


\section{REFERENCES}

1. A.C.S. van Heel

A lens producing pure coma

Physical II, 1, 1935 (p.62-70).

2. R. Kingslake and A.B. Simmons

A method of projecting star images having coma and astigmatism.

Josa, vol.23, 1933 (p. 282-288).

3. A.C.S van Heel

Coma

Nederlands Tijdschrift van Natuurkunde.

1935 (p.94-97). 